\title{
Structurization of poly mineral is highly concentrated poly disperse systems based on organic binders
}

\author{
Eduard Kotlyarsky ${ }^{1 *}$, Naum Uriev $^{2}$, Yury Vasilev ${ }^{1}$, Vladimir Kochnev ${ }^{1,2}$, Igor Sarichev $^{1}$ \\ ${ }^{1}$ Moscow Road-Road State Technical University (MADI), Department of Road-building materials, \\ 125319, Moscow, Leningrad Prospect, 64, Russia. \\ ${ }^{2}$ Russian academy of sciences A.N. Frumkin Institute of Physical chemistry and Electrochemistry \\ RAS, 199071, Moscow, Leninsky prospect, 31, Russia
}

\begin{abstract}
At present, the quality of bitumen-mineral mixtures both on the territory of the Russian Federation and abroad is estimated by a set of empirical methods based on many years of experience in the use of asphalt in road construction. In different years, numerous attempts were made to theoretically substantiate the basic patterns of material behavior under the influence of various factors. The article describes the solution of this problem using the basic provisions of phys-chemical mechanics of highly concentrated disperse systems taking into account the specifics of contact interactions. As a result of researches the quantitative correlations of indicators of physical-mechanical and structural-rheological properties forming at compaction of mixtures mixes, quantitative interconnections between formative and Destructive factors with structural-mechanical and construction-technical properties of asphalt in road coverings. It has been established that the changes in the structure of asphalt have physical-chemical nature and are reliably described taking into account the basic provisions of phys-chemical mechanics and theories of contact interactions.
\end{abstract}

\section{Introduction}

Asphalt concrete has complex rheological properties which define both the technology of work and operational reliability of the created road constructions. In different countries there is a methodical basis for quality assessment of bitumen-mineral mixtures.

At different points in time, numerous attempts were made to establish basic patterns of material behavior under the influence of various technological and operational factors. This allowed to establish that changes in the ratios of components and their qualitative conditions determines their physical and mechanical characteristics. It is proven that additive properties of asphalt mixes and asphalt are defined by "macro", "meso", and, in particular, "micro-structure", properties of which depend on physical and chemical phenomena on the boundary of Section Binder (bitumen) - stone material.

\footnotetext{
Corresponding author: eco46@mail.ru
} 


\section{Literature Review}

Professor I.A. Rybev [1] proposed the theory of building conglomerates, Professor V.P. Selyatin[2] developed the polistructure theory. Professor M.I. Volkov and his students investigated the processes of directional control of asphalt properties by regulating the properties of binder in the contact zone between the mineral particles.

Different theoretical positions of structure formation asphalt concrete were proposed by different authors at different times. According to the representations of P.V. Sakharov [3], I.A. Rybev [1], M.I. Volkov [4], N.V. Goryshev [5], I.V. Korolev [6], I.N. Kovalev [7], etc. Optimal structures are formed at a certain optimal content of organic binder.

Asphalt mixtures and asphalt concrete used for road pavement are highly concentrated dispersion systems. These systems are characterized as applied to bitumen-mineral mixtures which have a highly developed interphase surface $S$ with high concentrations of dispersion phase in liquid and gas dispersion states.

\section{Materials and methods}

The creation of optimal conditions for the formation of microstructural contact links in highly concentrated polimineral polidispersion systems contributes significantly to the formation of asphalt mixes with the necessary technological properties and asphalt with the required transport performance. Quantitative criteria of estimation of a condition of structure of a material for road asphalt and asphalt mixes in the normative-technical base have not been regulated up until now.

This task is theoretically solved using the basic provisions of the physicochemical mechanics of highly concentrated dispersion systems taking into account the specifics of contact interactions.

It is established, that at high levels of polimineral and polidispersion filler particles with structural shells formed on them with highly structured bitumen interact with each other on micro contact platforms that have the diameter of di [1]. This promotes the formation of new coagulating structures with grains of active thin-disperse mineral fillers acting as centers of formation. As the temperature increases, the interphase contact interaction decreases. On the basis of the works of P.A. Rebinder, E.D. Schukin, L.Y. Margolis [8] It is suggested to use dependence of durability of porous bodies, taking into account summation of strength of elementary contacts:

$$
P_{m}=k_{1} \cdot F_{c} \cdot n^{2 / 3}=k_{2} \cdot \varphi \cdot S_{y d}^{2}=k_{3} \cdot F_{c} \cdot \varphi \cdot d_{э \kappa \varepsilon}{ }^{2}
$$

Where: $\boldsymbol{P}_{\boldsymbol{m}}-$ The limiting shear stress $\boldsymbol{F}_{\boldsymbol{c}}$ - the average coupling force in the contact between the particles $\boldsymbol{n}$ - the average number of contacts between particles in the volume unit $\left(\mathrm{m}^{3}\right), \boldsymbol{\varphi}$-the relative density of the structure, $S$ - specific surface of particles of dispersed phase of highly concentrated dispersion system, $\boldsymbol{d}_{\boldsymbol{i}}$-the characteristic size of these particles.

\section{Research results}

Modern equipment allows for precise estimation of the limiting voltage of shift $\boldsymbol{P}_{\boldsymbol{m}}$ of highly concentrated dispersion systems.

To determine the average number of contacts between dispersed particles in a volume unit, it is possible to use an empirical expression obtained earlier by authors in coauthorship with V.N. Finashin and V.E. Chernomaz [9]: 


$$
n=6.7373 \cdot 10^{10} \cdot e^{\left(5.44 \varphi_{i}\right)} \cdot d_{\text {эк }}^{2}
$$

Where: $\boldsymbol{n}$ - the average number of contacts between particles in the volume unit, $\boldsymbol{\varphi}_{i}$ relative density of the compacted (sealed) asphalt mixture, $\boldsymbol{e}$-base of natural logarithms, $\boldsymbol{d}$ typical size of particles in the mineral part of asphalt mixture.

The conditions of interaction between particles of the dispersed phase depend on the distance between them. The true strength of a single contact depends on the conditions of its formation. They define a fixed even thickness of a layer of a liquid (bitumen) phase binder between minerals. First of all it is important for the formation of a microcontact structure with thin dispersion particles. This is confirmed by various aspects of the theory of strength of porous bodies, offered in the works of E.D. Yakhnin, A.F. Polak, N.B. Uriev, etc. $[10,11,12]$.

The difficulty of establishing the functional dependence of the shear stress limit, the average coupling force in contact between the particles and the average number of contacts per unit of volume $\left(\boldsymbol{P}_{\boldsymbol{m}}\right.$ from $\boldsymbol{F}_{\boldsymbol{c}}$ and $\left.\boldsymbol{n}\right)$ is that it was theoretically substantiated in due time and experimentally confirmed for monodisperse monomineral spherical particles [9]. In real asphalt mixes it is necessary to additionally consider heterogeneity connected with polidispersion and polimineral characteristics of mineral particles of the random irregular form.

The average coupling force in the contact between the particles $\left(\boldsymbol{F}_{\boldsymbol{c}}\right)$ can be determined by experimentally obtained values of the shear stress limit and the known geometric parameters of the investigated dispersion system using the converted formula of Prof. E.D. Yakh$\operatorname{nin}[13]$ :

$$
F_{c}=P_{m} \cdot d_{э \kappa в}^{2} \cdot \rho^{\prime} \cdot \frac{\left(\rho-\rho_{i}\right) \cdot\left(\rho-\rho_{p}\right)}{\rho^{2}\left(\rho_{i}-\rho_{p}\right)}
$$

Given that $\varphi_{\mathrm{i}}=\rho_{\mathrm{i}} / \rho$ and $\varphi_{\mathrm{p}}=\rho_{\mathrm{p}} / \rho$ expression (3) can be represented as:

$$
F_{c}=P_{m} \cdot d_{э \kappa s}^{2} \cdot \rho^{\prime} \cdot \frac{\rho^{\prime}}{\rho_{n}} \cdot \frac{\left(1-\varphi_{i}\right) \cdot\left(1-\varphi_{p}\right)}{\left(\varphi_{i}-\varphi_{\rho}\right)}
$$

Where: $\boldsymbol{\rho}$ ' - Density of the mineral part of asphalt, $\mathrm{kg} / \mathrm{m}^{3}, \boldsymbol{\rho}$-density of asphalt, $\boldsymbol{\rho}_{\boldsymbol{i}}-$ achieved as a result of technological influences density of asphalt, $\mathrm{kg} / \mathrm{m}^{3}, \rho_{\boldsymbol{p}}$-average density of unsealed asphalt mixture, $\mathrm{kg} / \mathrm{m} 3$, Which is determined experimentally and depends on the average density of the initial components, included in the composition of the asphalt mixture and their weight ratio, $\boldsymbol{d}$-equivalent particle size of the mineral part of the asphalt mixture $\varphi_{i}$-relative The density achieved as a result of technological influences on the asphalt mixture, $\varphi_{p}$ - the same as $\varphi_{i}$ but completely loose.

The average area of a single contact is determined by the condition of the additive strength of the elementary contact of the dispersed system (1):

The total area of elementary (single) contacts is obtained using the expression:

$$
\sum S_{i}=\frac{1}{k_{1}}=\left(\frac{F_{c}}{P_{m}}\right) \cdot n^{2 / 3}
$$

Then the average area of a single contact $\left(S_{i}\right)$ at their total number of n equals: 


$$
S_{i}=\frac{\sum S_{i}}{n}=\left(\frac{F_{c}}{P_{m}}\right) \cdot \frac{n^{2 / 3}}{n}=\left(\frac{F_{c}}{P_{m}}\right) \cdot n^{-1 / 3}
$$

The average size of a single contact can be found if the shape of its surface is known, which depends on the conditions of contact formation, the size and shape of mineral particles, the viscosity of bitumen and many other factors that are not always amenable to direct quantitative accounting. For simplification it is accepted that coagulation contact between particles is formed on a circular surface, in which case the size (or average diameter) of a single elementary contact will be equal to:

$$
d_{i}=\sqrt{\frac{4 \cdot S_{i}}{\pi}}
$$

The average strength of a single contact $\left(\boldsymbol{P}_{\boldsymbol{i}}\right)$ is determined from the following conditions:

$$
\boldsymbol{P}_{i}=\frac{\boldsymbol{F}_{c}}{\boldsymbol{S}_{i}}=\boldsymbol{P}_{m} \cdot \boldsymbol{n}^{2 / 3}
$$

S.J. Shalyt, N.V. Mikhailov and P.A. Rebinder [18] have shown that film from calcium soaps forms on particle of fillers, and the viscosity of bitumen increases under the exponential law as it approaching a particle from distance $\boldsymbol{h}_{\boldsymbol{0}}$ (Fig. 1).

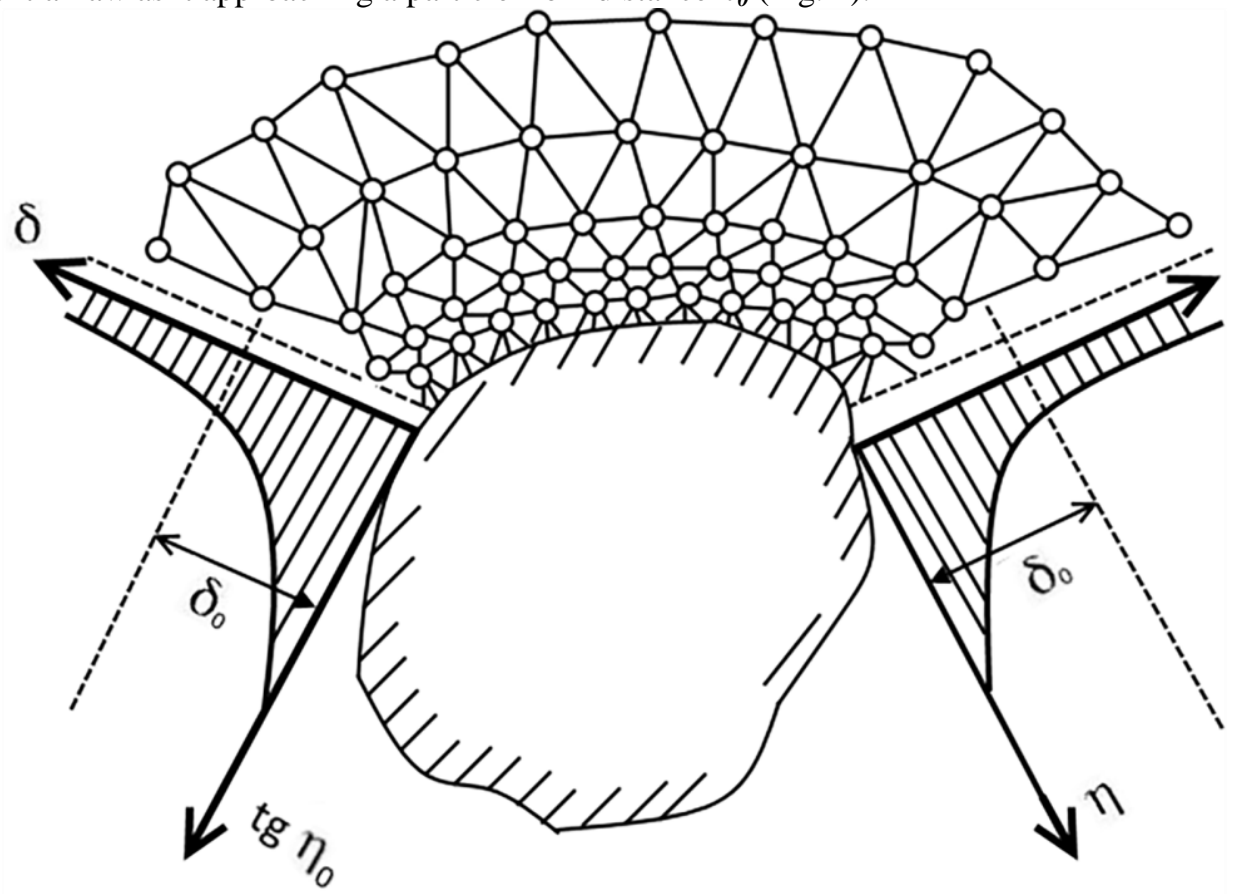

Fig. 1 structure of bitumen film in the contact zone of S.J. Shalyt, N.V. Mikhailov, P.A. Rebinder [18]. 
A.F Polak, B.V Deryagin, L.D. Landau [19] gave an explanation of the interaction in the zone of contact with energy positions (Fig. 2). At the same time the far coagulation distance $\boldsymbol{h}_{\boldsymbol{0}}$ (about $10^{-7} \mathrm{~m}$ ) and near coagulation distance (about $10^{-9} \mathrm{~m}$ ) are determined.

A similar qualitative picture of the interaction of bitumen and mineral particle leads in a number of Works Professor M.I. Volkov and I.V. Korolev [20]

All this suggests that the average bonding force in contact between particles is a quantitative estimate that characterizes the quality of coagulation links. This force depends on conditions of formation of single contacts under technological influences, adhesive properties of bitumen and stone materials. This parameter also allows to estimate results of influence on bitumen-mineral materials of external factors in the course of exploitation of a material in constructive layers of road pavements.

In laboratory, production and field conditions, at the "organic binders and asphalt concrete" laboratory of MADI, together with the laboratory of highly concentrated dispersed systems of RAS, was developed the methodology of quantitative estimation of the processes of formation of structures in asphalt mixes (at a technological stage) and destruction of the formed asphalt structures under the influence of operational factors. For this purpose, the shear stress limit $\left(\boldsymbol{P}_{\boldsymbol{m}}\right)$ was determined by experimental methods.

\section{Conclusions}

1. Directional regulation of the processes of forming the structure of asphalt mixtures and asphalt should be carried whilst taking into account the theory of formation of highly concentrated dispersion systems and the specifics of contact interactions.

2. Quantitative correlation of indicators of physical-mechanical and structural-rheological properties forming at sealing of highly porous bitumen of sand mixes and dense asphalt of different composition on various binding materials is established for materials with technological parameters of the sealing process.

3. It is established, that during sealing of asphalt mixes, the achievement of density of asphalt norms in constructive layers of road surfaces does not guarantee all required complex of physical and mechanical properties and structure of a material, which meet the operational requirements.

4. It can be assumed that the changes in the structure of asphalt are of physical and chemical nature and are reliably quantified according to the basic provisions of the operational factors and physicochemical mechanics and the theory of contact interactions when working on road surfaces.

The research was carried out within the scope of the subject: "Physicochemical dynamics of structured colloidal and nanodispersion systems and composite materials No. 01201355826" IFHJE RAS.

The work was performed using the equipment of the center of collective use of MADI (CKP MADI).

\section{Reference}

1. Rybev I.A. High school // Building Materials Science., 2003, $701 \mathrm{p}$

2. Solomatin V.I., Selyatin V.P. Elements of the general theory of composite materials // Construction and architecture. No. 8. 1980, p. 61-70.

3. Sakharov P.V. Methods of designing asphalt concrete mixes // Transport and roads of the city. No.12. 1935, p. 22-26.

4. Volkov M.I. Road building materials // Transport. 1965, p. 347-405.

5. Goryshev N.V. Asphalt concrete and other bitumen-mineral materials // Mojaysk-Terra. 1995, p. 176. 
6. Korolev I.V. Thesis for the academic degree. Structure and properties of the warm road asphalt. 1982.

7. Kovalev I.N. Activation technologies of road composite materials (scientific and practical basis). Monograph // Belarusian Encyclopedia. 2002, p. 334.

8. Rebinder P.A., Schukin E.D., Margolis L.Y. Report of the SA USSR No.154. 1964 696p.

9. Finashin V.N., Chernomaz V.E., Kotlyarsky E.V., Uriev N.B. In the collection of scientific works "ways of perfection of technology of production and improvement of quality of road-building materials " // Stages of formation of asphalt mixtures at their sealing, 1987,p.118-128

10. Yakhnin E.D. On the relationship between the strength of the dispersed structure and the forces of interaction between elements // Report of the SA USSR No. 178, 1968, 152 p.

11. Polak A.F. Hardening monomineral binder substances // Stroyizdat. 1966, 208 p.

12. Rebinder P.A.,Uriev N.B. The basic stages of formation and destruction of coagulation structures and their role in optimization of technological processes in the structured disperse systems // DAN USSR

13. Yakhnin E.D. Report of the SA USSR., No. 164., 1965, 1105 p.

14. Kotlyarsky E.V. Thesis for the academic degree. Improvement of durability of road pavements by optimizing the structure of asphalt, 2013

15. Kiruhin G.N., Kazarnovsky V.D. Development of normative base of calculation characteristics of asphalt // In the collection of articles and reports of the annual Scientific session of AIA.2010, 86-92 p.

16. Nikolsky U.E., Babak O.G., Starkov G.B., Gubach L.S. Methodical recommendations on evaluation of sdvigoustojchivosti asphalt. 2002, 20 p.

17. Gorelishev N.V., Goglidze V.M., Radovsky B.S., Suprun A.S. Methodical instructions on definition of unstandardized properties of asphalt at the course "Road-building materials". 1988, $30 \mathrm{p}$.

18. Shalyt S.J., Mikhailov N.V., Rebinder P.A. Effect of active filler and solvent on bitumen properties. No.19, 1957, 244-251 p.

19. Deryagin B.V, Landau L.D. Journal of Experimental and Theoretical physics. No.15, 1945, $663 \mathrm{p}$.

20. Korolev I.V. Ways of saving bitumen in road construction. 1986, 149 p. 